\title{
Cambio climático y migración en los pueblos indígenas de la provincia de Chimborazo - Ecuador
}

\begin{abstract}
(c) (i) (2)(2)
Climate change and migration in the indigenous peoples of the province of Chimborazo - Ecuador

Bolívar Urquizo Tenesaca. ${ }^{1}$

\begin{abstract}
.
DOI: https://doi.org/10.33262/concienciadigital.v4i1.2.1623

Introduction. Adaptation to climate change (CC) has become one of the transcendental objectives of the 2030 Agenda for Sustainable Development on a global scale. Thus, there is a need to identify gaps in the link between CC and migration in order to achieve greater stability between prevention, adaptation, mitigation, humanitarian response and development for CC victims. Objective. This article aims to establish a scenario on $\mathrm{CC}$ and migration in the indigenous peoples of the province of Chimborazo, Ecuador. Methodology. The study was based on a documentary review as a research method. Also, a large part of this report is a context of theoretical reflection, based on the convergence of academic and critical reading practices. Results. The results analyzed show that forced migration in the province of Chimborazo, Ecuador, is a consequence of environmental effects. The systemic link

\footnotetext{
${ }^{1}$ Ministerio de Relaciones Exteriores y Movilidad Humana, Consulado del Ecuador en Londres, Tercer Secretario/Vicecónsul, Londres, Reino Unido. email: bolivarurquizo@gmail.com ORCID ID: https://orcid.org/0000-0002-6320-5850
} 
between CC, disasters and forced migration is increasingly recognized as a strategy for survival, growth and adaptation of human beings. Conclusion. Understanding the role of the environment in the migratory processes of the indigenous peoples of the province of Chimborazo, Ecuador, requires the analysis of the conditions of vulnerability of the population to the effects of $\mathrm{CC}$, as well as the examination of their coping strategies, and to advance in the enactment of public policies in an effective manner and without leaving anyone behind.

Keywords: Climate change, sustainable development, environment, migration, indigenous peoples.

\section{Resumen.}

Introducción. La adaptación al cambio climático (CC) se ha convertido en uno de los transcendentales objetivos de la Agenda 2030 para el Desarrollo sostenible a escala mundial. Surgiendo así la necesidad de identificar vacíos en el vínculo entre CC y migración para conseguir una mayor estabilidad entre prevención, adaptación, mitigación, respuesta humanitaria y desarrollo para las víctimas del CC. Objetivo. A través del presente artículo se pretende establecer un escenario sobre el CC y migración en los pueblos indígenas de la provincia de Chimborazo, Ecuador. Metodología. El estudio se basó en una revisión documentaria como método de investigación. También, una gran parte de este informe es de contexto de reflexión teórica, asentada en la convergencia de prácticas de lectura académica y crítica. Resultados. Los resultados analizados, expresan que la migración forzosa la provincia de Chimborazo, Ecuador, consigue ser una consecuencia de los efectos ambientales. El vínculo sistémico entre el CC, los desastres y la migración obligada cada vez asume un mayor nivel de reconocimiento, pues es una estrategia de supervivencia, crecimiento y adaptación del ser humano. Conclusión. La comprensión del rol del medio ambiente en los procesos migratorios de los pueblos indígenas de la provincia de Chimborazo, Ecuador, exige el análisis de las condiciones de vulnerabilidad de la población frente a los efectos del $\mathrm{CC}$, así como el examen de sus estrategias de afrontamiento, y avanzar en la promulgación de políticas públicas de manera efectiva y sin dejar a nadie atrás.

Palabras claves: Cambio climático, desarrollo sostenible, medio ambiente, migración, pueblos indígenas.

\section{Introducción.}

La problemática generada por el cambio climático (CC) es multidimensional, ya que afecta a la flora, a la fauna y notablemente a colectividades consideradas ancestrales (Hernández, 
2021). Esta situación constituye un agravio ambiental, social y de acción colectiva (Sandoval-Díaz, et al., 2021). Por tanto, la lucha contra el fenómeno se ha inscrito poco a poco en la agenda global y esta, a su vez, ha tenido repercusiones en las políticas internas de varios Estados alrededor del mundo (Portador-García, 2020). Así, según Vázquez (2020) se ha puesto en marcha un proceso de gobernanza multilateral. Desde sus inicios, los debates en torno al CC se han focalizado en aspectos científicos o económicos; sin embargo, pese a los esfuerzos, la emergencia del CC como problema universal y el papel que juegan los bosques como estrategia de mitigación del CC coloca a los pueblos indígenas en un papel protagónico, en un mundo globalizado (Muyulema, 2018; Chowdhry \& Ruiz, 2019; Michel, 2020).

Si bien la migración consigue considerarse como una estrategia sistémica de adaptación a la que se ven forzadas diversas personas por los efectos a largo plazo del CC, igualmente puede tornar a las mujeres y los hombres indígenas más marginados y vulnerables a la discriminación, la detrimento de identidad, la explotación y a otros riesgos económicos, sociales y ambientales en sus lugares de destino, frente a distintos grupos de la sociedad, incluidas las personas que sufren pobreza extrema (Cadilhac, Torres, Calles, Vanacker, \& Calderón, 2017). La falta de reconocimiento de sus competencias laborales tradicionales, la formación académica deficiente y la apatía por sus culturas y medios de vida son algunos de los inconvenientes a que se afrontan para conseguir un trabajo decente cuando la población indígena se ha visto en la necesidad de migrar (Urquizo-Tenesaca \& Muyulema-Allaica, 2019). Una investigación reciente de la Organización Internacional del Trabajo (OIT, 2018) evalúo el sometimiento del salario mínimo en diez economías de mercados emergentes y en desarrollo, observando que la mano de obra de las comunidades indígenas está crecidamente expuesta a mayores brechas de inobservancia que otros grupos de clase obrera, y emplaza a las mujeres indígenas en un contexto de desventaja agravada.

Un sinnúmero mujeres y hombres indígenas en Ecuador encuentran trabajo en la economía informal y participan en actividades como el trabajo estacional y ocasional en explotaciones agrícolas, plantaciones, obras de construcción en compañías informales, en la venta ambulante como principal actividad económica en el contexto de la informalidad o como trabajadores domésticos (Michel, 2020). Estos trabajos tienden a ser peligrosos o precarios y a menudo se caracterizan por la segregación laboral, salarios bajos, falta de afiliación al sistema de seguridad social, modalidades contractuales deficientes y exposición a altos niveles de riesgos para la seguridad y salud, así como por situaciones de trabajo forzoso u obligatorio (Abarca, et al., 2019; Toulkeridis, et al., 2020)

En aras de enfrentar esta problemática, el Estado ecuatoriano presentó en 2012 una iniciativa denominada Estrategia Nacional de Cambio Climático (ENCC) que es la consecuencia de un esfuerzo inmutable liderado y facilitado por el Ministerio del Ambiente de Ecuador (MAE), a quien se le delegó la función de liderar con éxito la gestión del CC a escala nacional, como Autoridad Ambiental Nacional (Cadilhac, et al., 2017). Esta propuesta pretendía gobernar y 
dictar de manera sistemática y regularizada las diversas acciones y medidas que el Ecuador precisa impulsar para preparar a la Nación a afrontar los acontecimientos extremos climáticos de mayor frecuencia e intensidad; que tendrán sin duda afectaciones significativas en todos los sectores económicos del país (Muyulema, 2018). Pero sin duda, el CC es una complicación latente a nivel global, convirtiéndose en indudables desafíos ambientales y cambios significativos, muchos de ellos soportando una reacción irreversible, es así que los efectos concurridos en Ecuador se evidencian en el conocimiento biofísico del territorio, como en el deshielo de los nevados andinos, en el incremento de la temperatura, inundaciones, sequías, acrecentamiento del nivel del mar, incidencias negativas de las actividades productivas y en la biodiversidad, entre otros.

Existe un debate de modo consistente a nivel científico y en los procesos de toma de decisiones hacia la delineación de políticas internacionales que generen compromiso en todos los países que han ratificado su acuerdo en el contexto de la Convención Marco de las Naciones Unidas sobre CC. Este es el sitio de concertación en donde el Ecuador y otros países participan para precisar los pactos y normativas internacionales hacia reducir de forma integral las emisiones de gases de efecto invernadero (GEI) que constituyen los principales orígenes de la problemática a escala mundial, y está a su vez afecta con mayor intensidad a los países en vías de desarrollo y en países en desarrollo (Sánchez-Macías, et al., 2020). Adicionalmente, la Convención orienta el accionar de los gobiernos en la ejecución de medidas de adaptación frente al aumento de la temperatura global del planeta (Vázquez, 2020). Según modelos de proyección, la temperatura en Ecuador podría aumentar en $2^{\circ} \mathrm{C}$, con excepción de la región Amazónica e Insular, pues estas expondrían incrementos superiores (MAE, 2019). Los resultados de los últimos informes resaltan que de las fuentes de emisiones de GEI y particularmente $\mathrm{CO}_{2}$ en el país son los siguientes: el sector energético con un 44,49\%, continuo del uso de suelo cambio de uso de suelo y silvicultura (USCUSS) con el 30,02\%, agricultura con el 18,03\%. Mientras que los sectores de residuos y procesos industriales reconocen un $4,16 \%$ y un $3,30 \%$, correspondientemente (MAE, 2016).

La provincia de Chimborazo se encuentra ubicada en el centro del Ecuador entre la cordillera oriental y occidental. Su ubicación permite la formación de 13 ecosistemas y de una diversidad de pisos climáticos que permiten el desarrollo de actividades productivas asentadas principalmente en el valle central de la provincia (Pucha-Medina, et al., 2019). Esta diversidad climática, permite el cultivo de productos de zonas frías y de zonas tropicales (Peralta, Mejía, \& Gavidia, 2020). No obstante, los cambios que se dan en las zonas rurales, son profundos, y están sujetos a los efectos combinados de globalización y localización (Muyulema-Allaica, et al., 2019). Es así que gran parte del crecimiento urbano es exógeno puesto que se debe a los numerosos flujos de migración categórica del campo a la ciudad, generando procesos de inestabilidad, sobre todo en el ámbito laboral, una de las causas es la migración (interna e internacional) (Urquizo-Tenesaca \& Muyulema-Allaica, 2019). 
En Chimborazo, la población rural considerada la más pobre vive en espacios expuestos y marginales, y en circunstancias que los hacen estrechamente vulnerables a los impactos nocivos del CC (Peralta, Mejía, \& Gavidia, 2020). Para estas personas, aún las mínimas permutaciones en el clima consiguen tener un impacto innegablemente desastroso en sus vidas y medios de sustento (Mejía, Mejía, Veloz, \& Muquinche, 2019). Bajo los argumentos anteriores, las consecuencias son muy difíciles para los agricultores, puesto que su medio de subsistencia está ubicado en ambientes frágiles, donde se esperan grandes cambios en su productividad, pues estos cultivadores dependen de sus labranzas (Fonseca, Haro, Inca, \& Abdo, 2019).

En casos de pérdidas en los eslabones de producción la actividad agropecuaria se ha desplazado a la actividad agrícola asalariada bajo un proceso migratorio más o menos permanente y este supone estar obligado a adaptarse y a modificar sus formas de vida e incluso la organización social (Cordero, et al., 2017; Toulkeridis, et al., 2020).

Por lo antes expuesto, es necesario establecer un escenario sobre el CC y migración en los pueblos indígenas de la provincia de Chimborazo, Ecuador, con el fin de contribuir en la generación de nuevas políticas agrícolas y productivas que permitan a la población enfrentar los cambios climáticos en la provincia.

\section{Metodología.}

El estudio se ha centrado en la provincia de Chimborazo, ubicada en el centro de la región interandina del Ecuador, conocida como la Sultana de los Andes, debido a que alrededor de ella se encuentran grandes cumbres, el volcán Chimborazo, Carihuayrazo, El Altar, el Igualata, el Tungurahua, el Sangay, varias de estas cumbres comparten con otras provincias. Administrativamente tiene 10 cantones (Alausí, Chambo, Cumandá, Penipe, Colta, Riobamba, Guamote, Chunchi, Pallatanga y Guano) y 45 parroquias rurales. Su ubicación permite la formación de 13 ecosistemas y de una diversidad de pisos climáticos que permiten el desarrollo de actividades productivas asentadas principalmente en el valle central de la provincia.

El acceso hacia la provincia se lo puede realizar por vías de primer orden, la Panamericana, por la región interandina o sierra, desde la ciudad de Quito y Balbanera, por la región Costa, y, desde la ciudad de Guayaquil.

Este estudio se basa en la revisión documentaria como método de investigación. Adicionalmente, se ha revisado documentos informes sobre $\mathrm{CC}$ y migración en los pueblos indígenas de la provincia de Chimborazo, Ecuador de diferentes campos de estudios con perspectivas científicos, antropólogos, históricos y de ciencia política. También, una gran parte de este informe es de contexto de reflexión teórica, basadas en las lecturas críticas de ideas sobre esta relación. 


\section{Resultados.}

\section{Cambio Climático}

Por CC se entiende a todo cambio de clima imputado directa o indirectamente a la actividad humana que altera constantemente la composición de la atmósfera mundial y que se adiciona a la variabilidad natural del clima observada durante un número significativo de periodos de tiempo comparables (Chowdhry \& Ruiz, 2019). Se originan a diversas escalas de tiempo; los parámetros climáticos, como temperatura, precipitación, nubosidad, entre otros, son los que se alteran debido a orígenes naturales y/o actividades humanas (Vázquez, 2020). Las evidencias perceptibles de este cambio son un aumento de la temperatura global por un incremento del efecto invernadero, cambios en el nivel del agua de los mares, deshielos polares, glaciares, entre otros (Toulkeridis, et al., 2020). Todas estas transformaciones generan sequias, incendios forestales, olas de calor, aluviones, lluvias torrenciales, huracanes, entre otros desastres naturales (Cordero, Texidó, Modo, \& Mach, 2017).

El panorama de incertidumbre de América Latina frente al CC junto con la desigualdad o el consumo insostenible de los recursos naturales, es significativo, pese a mínimas emisiones de GEI, contrastadas con regiones de gran potestad económica y desarrollo industrial y tecnológico (Sánchez-Macías, et al., 2020). Según informe del Programa de las Naciones Unidas para el Medio Ambiente (PNUMA, 2016), la región de América Latina y el Caribe generan el 5.0\% de las emisiones globales de GEI. No obstante, según Cáceres-Arteaga \& D. Lane (2020) es factible recalcar, que entre las transcendentales fuentes de emisiones de GEI de América Latina constan el sector energético (46.0\%), la agricultura (23.0\%), cambio de uso de suelo y silvicultura $(19.0 \%)$, desechos (6.0\%) y procesos industriales $(4.0 \%)$.

Los trastornos del calentamiento global ya se están percibiendo en territorios de América Latina, por lo que debería tomar acciones o buscar adaptarse a la situación climática, recalcando que la generalidad de países de la región se dedica a actividades agro-productivas (Muyulema-Allaica, Canga-Castillo, Pucha-Medina, \& Espinosa-Ruiz, 2019). En ese sentido Conopoima (2021); Sandoval-Díaz, et al., (2021) las huellas del CC sobre América Latina y el Caribe para el 2020 estuvieron leves, puesto que ha experimentado una mejora considerable en la calidad del aire debido a que las medidas de distanciamiento social para reducir la propagación de la enfermedad del coronavirus (COVID-19) que han hecho que las personas permanezcan en sus domicilios y las empresas busquen nuevas formas de operar, pero proyecciones para el 2050 , advierten que la temperatura se aumentará en un $1,5^{\circ} \mathrm{C}$ a $2^{\circ} \mathrm{C}$, tomando como referencia la temperatura actual. En este contexto Álvarez, et al., (2020) señalan que en las últimas décadas las consecuencias negativas en los sistemas naturales son atribuidos a la relación holista de la acción humana - clima. En donde los escurrimientos presentan un mayor intervalo se sugiere valorar in situ los recursos naturales y establecer acciones de manejo. 


\section{Cambio Climático en el Ecuador}

Ecuador, es un país de origen andino, situado al noreste de América del Sur, contiguo al norte con Colombia, al sur y al este con Perú y al oeste con el Océano Pacifico. El país cuenta con cuatro regiones naturales: Costa, Sierra, Oriental y región Insular. El territorio posee una alta diversidad biológica, la más compacta del mundo (Urquizo-Tenesaca \& Muyulema-Allaica, 2019). Por lo cual, Ecuador es un país pequeño en extensión territorial, y consigue ser un estado vulnerable ante los impactos negativos del CC, considerando su condición económica se basa principalmente en la agricultura, la minería y la pesca (Cadilhac, et al., 2017).

El clima ecuatoriano está explícito por dos estaciones: cálida y fría o también distinguidas como verano e invierno, por derivación de su ubicación en la línea ecuatorial y su influencia de la humedad procedente de la región Oriental (Toulkeridis, et al., 2020). Por tal moción, es preciso destacar la vulnerabilidad del país frente a posibles alteraciones producidas por el CC, surgido por el aumento de la temperatura global debido a la presencia de GEI que impiden que la radiación emitida por la tierra se disipe y, por lo tanto, favorece que se mantengan atrapada en la atmosfera (Muyulema-Allaica, Canga-Castillo, Pucha-Medina, \& Espinosa-Ruiz, 2019). Entre las principales causas se encuentran: el aumento mismo de los GEI, excesivo uso de fertilizantes y producción de residuos, quema de combustibles fósiles, junto a la deforestación de bosques y selvas (Portador-García, 2020; Cáceres-Arteaga \& D. Lane, 2020).

Los efectos en Ecuador se demuestran en la percepción biofísica del territorio, como en el deshielo de los nevados andinos, el incremento de la temperatura, sequías, inundaciones, aumento del nivel del mar, deslizamientos en terrenos cultivables, pérdidas en la producción y rendimiento de cultivos, amenaza a la seguridad alimentaria y la biodiversidad, entre otros (Chowdhry \& Ruiz, 2019; Sandoval-Díaz, Cuadra-Martínez, Orellana-Fonseca, \& SandovalObando, 2021). Sin embargo, surgen disímiles expectativas para establecer mejores prácticas agrícolas que compongan los aspectos ambientales, económicos y sociales, excitando la sostenibilidad en la producción agrícola (Cáceres-Arteaga \& D. Lane, 2020). Dentro de este contexto, Peralta, Mejía, \& Gavidia (2020) comentan que los GEI establecen factores transcendentales para el CC radicado en el efecto de su potencial de calentamiento global (Global Warming Potential - GWP). El aumento de los GEI está ligado a las actividades ejecutadas por los sectores económicos, como es el caso del sector agrícola que ha apoyado con el 24.0\% de las emisiones globales (Toulkeridis, et al., 2020). El IPCC (2015) reportó que las emisiones derivadas por el sector agrícola a nivel mundial estuvieron de $11,76 \mathrm{Gt}$ $\mathrm{CO}_{2}$ eq. Para el caso de Ecuador, el MAE (2017) reporto que las emisiones en el año 2012 fueron de 14.512,88 $\mathrm{Gg}$ de $\mathrm{CO}_{2}$ eq que pertenecen a gases como el $\mathrm{CO}_{2}$, el $\mathrm{CH}_{4}$ y $\mathrm{N}_{2} \mathrm{O}$ procedentes de suelos agrícolas (46,37\%), fermentación entérica $(43,43 \%)$, cultivo de arroz (7,48\%), manejo de efluentes y estiércol $(2,34 \%)$ y la quema de residuos agrícolas $(0,39 \%)$. El aumento de estos GEI consigue estimular a graves alteraciones ecológicas y económicas, y al mismo tiempo conjetura cambios impredecibles en los sistemas climáticos. 


\section{Políticas sobre el cambio climático en Ecuador}

El Ecuador es uno de los 180 países firmantes del Acuerdo de París y, considerando que sus principales emisiones de $\mathrm{CO}_{2}$ no son representativas a escala global, la responsabilidad que el país asume con la protección del medio ambiente es meritorio, no solo a causa de ha suscrito la mayoría de los acuerdos internacionales en la materia, sino además por su desarrollo interno a nivel constitucional y legal en los últimos 15 años (Martínez-Moscoso, 2019).

Desde la expedición de su Constitución (2008), Ecuador reiteró su responsabilidad con la protección a la naturaleza, reconociéndola como sujeto de derechos, así como con el agua, a la que concedió la posición de derecho humano y fundamental, con un progreso de dos años a la resolución 64/292, del 28 de julio de 2010, de la Organización de las Naciones Unidas (ONU). Implícitamente registró en su texto constitucional el adeudo del Estado para acoger medidas de mitigación del CC, a través de la restricción de las emisiones de GEI, así como adoptando medidas de conservación de bosques y vegetación.

La propuesta de la nueva constituyente resultó novedosa en los núcleos articulares eferentes al CC, ya que circunscribió como obligación estatal la adopción de medidas proporcionadas y transversales para su mitigación, todo ello con la intención de restringir las emisiones de GEI, la deforestación y la contaminación del aire (Michel, 2020). En resultado, la norma ha forzado a que el Estado ecuatoriano, mediante la generación de políticas públicas, forje medidas para la preservación de los bosques y la vegetación y la protección de poblaciones vulnerables y personas de riesgo (Chowdhry \& Ruiz, 2019).

La adición de estos factores forjó un acaecimiento dinámico sobre el medio ambiente, ya que si bien es cierto se halla reconocido el derecho de la población a vivir a un medio ambiente sano y ecológicamente equilibrado (Martínez-Moscoso, 2019). La muestra bajo la visión biocentrista y el reconocimiento de la naturaleza como sujeto de derechos son los cuerpos normativos preceptos con sucesión a su entrada en vigor de la nueva Constitución, como la Ley Orgánica de Recursos Hídricos, Usos y Aprovechamiento del Agua de 2014 y el Código Orgánico del Ambiente de 2018, que son analizados como normas de referencia para la protección de los recursos hídricos y del CC (Muyulema-Allaica, Canga-Castillo, PuchaMedina, \& Espinosa-Ruiz, 2019; Cáceres-Arteaga \& D. Lane, 2020). De forma alineada a la misma, existe el Plan Nacional de Cambio Climático (PLANACC), como un soporte en la generación de información concreta por cada uno de los sectores vulnerables al fenómeno climático: ecosistemas, agua, agricultura y energía. Por otro lado, con el objeto de transversalizar la gestión a nivel territorial, la Subsecretaría de Cambio Climático (SCC) del MAE publicó la Guía Técnica para Incorporar el CC en la Planificación Local, que consiente de forma sistémica articular los esfuerzos con los Gobiernos Autónomos Descentralizados (GAD). No obstante, esta Cartera de Estado presentó el Reconocimiento Ecuatoriano Ambiental Carbono Neutral, herramienta que permitió robustecer el compromiso 
mancomunado entre el sector público y privado con el único fin de iniciar prácticas ambientalmente responsables en los sectores económicos, productivos, y de producción.

\section{Cambio Climático en Chimborazo}

La provincia de Chimborazo, considerada como una provincia eminentemente agrícola, así como una de las más pobres del Ecuador (Peralta, Mejía, \& Gavidia, 2020). Se estima que alrededor del $60 \%$ de la población es de origen indígena autodenominada kichwa, aprovisionada de territorios idóneos para las laborales agrícolas, los que al trascurrir el tiempo han ido disipando su fertilidad y el rendimiento ineludible para alcanzar niveles convenientes de rentabilidad, lo que ha ocasionado que las franjas agrícolas vayan ganando espacios invadiendo las zonas prioritarias de cobertura natural (Varea, 2020; Tuaza, Jonhson, \& McBurney, 2020).

Por otra parte, la pérdida de cobertura natural se manifiesta especialmente en el cambio del calendario agrícola, aumentando drásticamente el número de días lluviosos y disminuyendo el número de días secos, produciendo malestar a los agricultores debido a los detrimentos de cultivos ya sean por exceso o falta de precipitación (Tuaza, Jonhson, \& McBurney, 2020). La provincia de Chimborazo se enfrenta a tres problemas relacionados con la variabilidad del clima. El primero de ellos está relacionado a la cantidad y calidad de agua adecuados para la producción y procesos después de la cosecha; el segundo problema es que el aumento de la temperatura y el tercer problema es la irregularidad en la pluviosidad que ya ha generado reducciones en la producción y en ocasiones perdidas de la cosecha entera (Estrada \& SuárezDuque, 2020; Vivar \& Sánchez, 2020; Tuaza, Jonhson, \& McBurney, 2020).

Además, el nevado Chimborazo, ubicado en el extremo occidental de la provincia, es el volcán y la montaña más alta en el Ecuador, y el punto más distante del centro de la Tierra, se encuentra de $6.310 \mathrm{msnm}$, de cuyos deshielos da comienzo a vertientes sustanciales para los habitantes de las provincias de Tungurahua y Chimborazo, en los últimos 40 años, se observa la pérdida del $38 \%$ de su cubierta de hielo y cada vez son más visibles las zonas de tierra descubierta que transitan a ser ocupadas por líquenes y musgos, y por ende la principal afectación se visualiza en el suministro de agua (La Frenierre \& Mark, 2017).

\section{Migración en los pueblos indígenas a causa del Cambio Climático}

Existen disímiles definiciones acerca de la Migración, para Urquizo-Tenesaca \& MuyulemaAllaica (2019) es el desplazamiento geográfico de personas o grupos de personas, ordinariamente por causas económicas, sociales, ambientales o políticas. Para Pérez (2018) es el movimiento de una población hacia una región determinada interna o externa a su Estado de origen y tiene implicaciones para comunidades, hogares y personas. Por otro lado, Cordero, et al., (2017); Pérez (2018); Michel (2020); Hernández (2021) señalan que existe suficiente evidencia científica que advierte de forma enérgica los riesgos a los cuales se enfrenta la humanidad a causa del CC, atribuido directa o indirectamente a la actividad 
humana que altera la estructura de la atmósfera mundial y que se adiciona a la variabilidad natural del clima observada durante varios períodos de tiempo comparables.

Cadilhac, at al., (2017); Chowdhry \& Ruiz (2019); Michel (2020) exponen que la relación existente entre el CC y la Migración en Ecuador es compleja, y se reflexiona sobre las posibilidades de conocer sus implicaciones y su papel actual, para poder comprender la problemática. Para Cordero, et al., (2017); Urquizo-Tenesaca \& Muyulema-Allaica (2019) indican que la Migración en las 24 provincias del país, y en especial en la provincia de Chimborazo, podría diferenciarse como un impacto secundario y que una vez que se comprenda la problemática de esta relación, se puede analizar con mayor confianza. Peralta, Mejía, \& Gavidia, (2020) exponen las fluctuaciones climáticas en Chimborazo no solo impactan a la producción alimenticia, o al suministro de agua, así como a los patrones de infecciones y las enfermedades transmitidas por vectores, sino que también incentivan el desplazamiento geográfico de personas, exponiendo con ello el interés hacia tal problemática.

Las causas y las consecuencias del CC y Migración en los pueblos indígenas de la provincia de Chimborazo, están profundamente entrelazadas con patrones de desigualdad. Estas poblaciones que han contribuido menos a las causas del CC, como los pueblos y nacionalidades indígenas, son los más vulnerables a sus consecuencias y tienen la mayor probabilidad de que sus condiciones de vida empeoren.

Siguiendo su ciclo productivo los pueblos y nacionalidades indígenas de Chimborazo preparan el predio después de los meses de lluvia, que solían ser de enero a abril, siendo abril el período que recibe mayor precipitación, lo cual abona los suelos (disuelve minerales) y los "ablanda", por infiltración en suelos franco arenosos, pero en los franco arcillosos los endurece por la erosión hídrica laminar, en los arenosos los preparan en junio y julio, para finalmente sembrarlos desde agosto hasta diciembre, pero ahora la consecuencias del CC es precisamente la variabilidad climática, con manifestaciones cada vez más intensas y frecuentes, lo que confunde la planificación de las actividades agrícolas (Logroño-Rodríguez, et al., 2020; Chuiza-Rojas, Haro-Velasteguí, \& Brito-Moína, 2021).

La situación señalada anteriormente no solamente a impactados sustancialmente en la participación del sector a la actividad económica total de la provincia de Chimborazo, sino además afectara a los recursos con que cuente el país, el desarrollo del sector, abandono de campos de cultivo, y la subsecuente asignación presupuestaria (Sánchez-Macías, et al., 2020). Su principal hito ha sido el aumento de los flujos migratorios rurales hacia las principales ciudades de Ecuador como hacia el extranjero, siendo su principal receptor la Unión Americana (Michel, 2020). Los flujos migratorios internos y externos, inducen de forma dinámica en las comunidades rurales (pueblos indígenas) y está vinculado con la búsqueda de mejores condiciones de vida (estrategias de supervivencia o búsqueda oportunidades laborales), pero también se hallan motivadas por situaciones políticas, 
religiosas, raciales o vinculadas con catástrofes climáticas y con condiciones cambiantes de concentración poblacional, distribución de los recursos materiales y culturales (Vivar \& Sánchez, 2020; Peralta, Mejía, \& Gavidia, 2020). Es así como la migración transmuta la unidad de producción agrícola de sus comunidades de origen, y con ello la conservación de una actividad agro-productiva que no siendo rentable sigue preservándose en las comunidades agrícolas de la provincia.

Algunos de los cantones de Chimborazo, lugar de origen de los migrantes indígenas kichwa, participaron en la migración internacional, dirigido fundamentalmente a Estados Unidos $(43,61 \%)$, España (35,09\%) e Italia (7,20\%); sin embargo, la gran mayoría de los kichwa de esta provincia, que contiene la mayor densidad indígena del Ecuador, opto a la migración interna, exclusivamente para actividades de corte, alza y transporte de zafra (cosecha de caña de azúcar) en la costa, y comercio informal o venta ambulante, particularmente en ciudades grandes como: Guayaquil y Quito (Muyulema-Allaica, et al., 2019; Estrada \& Suárez-Duque, 2020).

Por otro lado, llama la atención que tanto hombres como mujeres que participaron en la migración interna y que pertenecen a las comunidades indígenas kichwa de la provincia de Chimborazo trabajen, lo que implica, por lo general, una carga adicional para ellas, pues conjuntamente suelen encargarse de los quehaceres domésticos del hogar y cuidado de los/as niños/as (Tuaza, 2019). Al mismo tiempo, se evidencia que las familias, tienen una considerable cantidad de hijos/as (4 hijos/as en promedio) que están involucrados en los trabajos desde una prematura edad (Tuaza, Jonhson, \& McBurney, 2020). El escenario normal de las familias migrantes indígenas es que ambos jefes de familia trabajan y que los hijos/as de la familia nuclear se implican en el trabajo, en la mesura que sus tareas escolares lo permiten (Abarca, et al., 2019). Se puede marcar una permanente tensión de los adolescentes por asistir a la escuela o al colegio, y las insuficiencias de la familia, y de ellos mismo por ganarse decentemente un ingreso económico con su trabajo (Izurieta, Atiencia, Fernández, \& Avalos, 2018). La mayor parte de las familias combinan, por lo usual, al menos dos trabajos: a menudo la venta en un local con informal o ambulante. Bajo este escenario, son escasas las parejas que se dedican a la misma actividad: así, diversas familias se dedican a la venta de frutas y verduras en sus locales, otras familias se concentran casi por completo, a la venta ambulante de gafas, y terceras se ocupan por vender ropa en locales generalmente arrendados (Varea, 2020; Sandoval-Díaz, et al., 2021). Pero, más allá de los negocios distinguidos en la provincia de Chimborazo, Ecuador, como informales, los indígenas jóvenes laboran en el sector formal, hay educadores en escuelas y colegios; odontólogos, médicos y abogados con consultorios propios, e ingenieros en ramas técnicas y administrativas.

Si bien, la migración forzosa la provincia de Chimborazo, consigue ser una consecuencia armónica de los efectos ambientales, igualmente puede desencadenar diversos conflictos conexos con la competitividad de los recursos ya escasos. El vínculo existente entre el CC, 
los desastres y la migración cada vez asume un mayor reconocimiento, pues se consagra como una estrategia de supervivencia y adaptación del ser humano. Bajo este contexto, es importante diseñar políticas y regulaciones. Ello se consigue con el desarrollo de las bases, el impulso de planes y programas de desarrollo productivo para pequeños agricultores, y la disminución de la pobreza y la dinamización del empleo rural no agrícola.

\section{Discusión}

La adaptación al CC se ha convertido en uno de los trascendentales objetivos para alcanzar el Desarrollo sostenible a escala mundial. En la actualidad residimos ante un panorama estrictamente complejo, la globalización marca día con día la línea base para llevar a cabo acciones que relacional lo ambiental, económico, social, político, y el personal (Muyulema, 2018). La sociedad se conecta por diversas formas y en diferentes dimensiones, sin embargo, tal vez no sean interacciones directas (Vázquez, 2020). Así, si a la interacción entre el CC, y la Migración, le agregamos la variable globalización, se estaría influyendo significativamente, no solo en las personas que son afectadas directamente por los estragos de este fenómeno migratorio, sino a toda la sociedad la cual debe, junto con el gobierno, articular esfuerzos para que esos efectos no trasciendan a mayores dimensiones y poder remediar las incidentes que se puedan presentar, esto, con el propósito de preservar vidas humanas (Muyulema-Allaica, Pucha-Medina, Espinosa-Ruiz, \& Urquizo-Tenesaca, 2019).

La relación existente entre Migración y CC no es un tema nuevo, ya que se han presenciado innegables circunstancias en el pasado, y en los últimos años diversas investigaciones han venido anunciando una preocupación latente y su relevancia se halla en aumento, debido a que cada vez es más potente la ocurrencia de desastres naturales y cambios considerables en la temperatura global, y por consecuente esto acarrea a que más individuos y en especial los pueblos rurales se vean afectados y obligados a adaptarse a diferentes acontecimientos mediante la migración externa y la interna.

Surgiendo de esta forma el término "Migración por Cambio Climático", la cual en concordancia con Cordero (2017) es aquel fenómeno que puede correlacionarse a cualquier tipo de cambio en el clima, a modo de desastres naturales, incremento de la temperatura o aluviones, sequias, entre otros. Dichos desplazamientos logran ser estacionales o permanentes y directos, como en el caso de inundaciones, o indirectos, como en el caso de incrementos de temperatura, lo cual consigue causar desertificación y el menoscabo de tierras cultivables y consiguientemente, destruir el sustento económico de la población afectada (Ferro, 2016; Sandoval-Díaz, et al., 2021). En Ecuador, son escasos los estudios realizados, entre ellos Cordero (2017); Cadilhac, at al., (2017); Michel (2020) exponen que las expresiones más significativas del fenómeno del CC, de forma usual y para el país, son: el incremento de la temperatura, la disminución clara de los recursos hídricos disponibles, la mayor periodicidad de fenómenos climáticos extremos, desgaste de biodiversidad, 
permutaciones en la agricultura y la cobertura vegetal, amenazas a los ecosistemas marinos, efectos sociales como el fenómeno de migración y los daños a la salud, entre otros.

De acuerdo con las evidencias recabadas, reflexionadas y analizadas a nivel nacional e internacional, se consigue deducir que la migración como una afectación motivada por el CC ya es una realidad y que cada vez el número de afectados ha crecido en ascendentes niveles, con lo cual es importante no solo cuantificar de forma integral los efectos e impactos, sino además diseñar medidas y acciones concretas que permitan a la sociedad estar al corriente sobre cómo proceder ante este desafío y con ellos poder salvaguardar vidas humanas, minimizar los impactos al ambiente y resguardar la infraestructura.

La agenda política de los pueblos indígenas de Ecuador está enfocada especialmente en la salvaguardia de sus territorios y de la vida (Varea, 2020). El tema del CC y del fenómeno migratorio ingresa intrínsecamente en este marco general (Michel, 2020). Las soluciones propuestitas desde la practica diplomática son respetar enérgicamente la vida de los pueblos indígenas, oír sus voces, entenderlos y comprenderlos, tomado en consideración que la migración de estos pueblos indígenas tiene al menos cuatro formas de obstaculizar el desarrollo: incrementa la presión sobre las infraestructuras y servicios urbanos, restringe el crecimiento económico, agranda los riesgos de conflictos y, entre los mismos migrantes, agrava los indicadores de salud, educativos y sociales.

La comprensión del rol del medio ambiente en los procesos migratorios en los pueblos indígenas de la provincia de Chimborazo, Ecuador, exige el análisis de las situaciones de vulnerabilidad de la población frente a los efectos del CC, así como el examen de sus estrategias de afrontamiento. Los efectos del CC cambian en función del contexto y la vulnerabilidad de la población y, sobre esto, el género y la clase social juegan un papel primordial, pues las acciones de las personas para soportar sus impactos varían en función de sus recursos y oportunidades.

\section{Conclusiones}

- El cambio climático (CC) una problemática donde se encuentra implícita la acción del hombre y donde todos estamos incluidos, puede minimizarse con las acciones que todos en conjuntos debemos realizar, desde repoblar con más plantas nuestro planeta, hasta optimizar el uso de los recursos que necesitamos para satisfacer nuestras propias necesidades.

- Todos formamos partes del CC, y todos debemos comprometernos para minimizarlos las emisiones que provoca, y así vislumbrar un mejor presente un cómodo futuro. No obstante, el tratamiento integral de la adaptación y mitigación frente al CC con perspectiva de migración en los pueblos indígenas en la provincia de Chimborazo continúa siendo un desafío para las instancias públicas y privadas en todo el Ecuador, considerando el marco político, normativo e institucional existente, como aspectos relevantes para fortalecer la política pública. 
- Debido a esto, se requiere una aproximación multidimensional que permita visibilizar y considerar, entre otras, las desigualdades estructurales para comprender las problemáticas, conflictos e interrelaciones que se derivan de este fenómeno. El manejo de un enfoque de derechos humanos es fundamental en su abordaje para garantizar la protección, el desarrollo y el bienestar de toda la población en su conjunto, y avanzar en la promulgación de políticas públicas de manera efectiva y sin dejar a nadie atrás.

\section{Referencias bibliográficas.}

Abarca, C. R., Santillán, M. E., Buenaño, B. C., Soria de Mesa, B. W., \& Abarca, S. V. (2019). Características del trabajo infantil en Riobamba. CSSN, 10(1), 60-68. doi:http://dx.doi.org/10.47244/cssn.Vol10.Iss1.178

Álvarez, P. A., Villavicencio, G. R., Ruiz, C. J., \& Santiago, P. A. (2020). Efecto del cambio climático a partir de los escenarios RCP 4.5 y 8.5 del año 2050 en el balance hídrico del Área Natural Protegida La Primavera, Jalisco, México. Áreas Naturales $\begin{array}{lll}\text { Protegidas Scripta, 6(1), } & \text { 53-74. }\end{array}$ doi:https://doi.org/10.18242/anpscripta.2020.06.06.01.0004

Cáceres-Arteaga, N., \& D. Lane, K. M. (2020). Agroecological Practices as a Climate Change Adaptation Mechanism in Four Highland Communities in Ecuador. Journal of Latin American Geography, 19(3), 47-73. doi:10.1353/lag.2020.0071

Cadilhac, L., Torres, R., Calles, J., Vanacker, V., \& Calderón, E. (2017). Desafíos para la investigación sobre el cambio climático en Ecuador. Neotropical Biodiversity, 3(1), 168-181. doi:10.1080/23766808.2017.1328247

Chowdhry, B. J., \& Ruiz, H. J. (2019). Entendiendo el cambio climático desde los glaciares andinos: Perspectivas desde Ecuador y Bolivia. Revista Harvard Review of Latin America, 1-15.

Chuiza-Rojas, M. R., Haro-Velasteguí, C. V., \& Brito-Moína, H. L. (2021). Identificación de las variables de proceso óptimas para la producción del almidón de papa china (Colocasia esculenta). Dominio de las Ciencias, 7(1), 837-848. doi:http://dx.doi.org/10.23857/dc.v7i1.168

Conopoima, M. Y. (2021). COVID-19 y medio ambiente: un llamado a la reflexión. Espíritu Emprendedor TES, 5(1), 29-44. doi:10.33970/eetes.v5.n1.2021.233

Constitución de la República del Ecuador. (2008). Constitución de la República del Ecuador. Montecristi : Asamblea Nacional Constituyente de Ecuador. 
Cordero, R., Texidó, E., Modo, V., \& Mach, E. (2017). Cuadernos Migratorios No. 8. Migraciones, Ambiente Cambio Climático: Estudios de Caso en América del Sur. Buenos Aires: Organización Internacional para las Migraciones (OIM). doi:https://environmentalmigration.iom.int/es/node/1298

Estrada, A. V., \& Suárez-Duque, D. (2020). Factores socioambientales que favorecen la conservación in situ de tubérculos alto andinos nativos en los cantones de Colta y Guamote en Chimborazo, Ecuador. Sociedad y Ambiente, 22, 72-96. doi:https://doi.org/10.31840/sya.vi22.2081

Ferro, M. (10 de 2016). El reconocimiento del Estatuto de Refugiado por la afectación a Derechos Fundamentales como consecuencia del Cambio Climático. Observatorio Medioambiental, 1(19). doi:http://dx.doi.org/10.5209/OBMD.54173

Fonseca, G., Haro, J., Inca, A., \& Abdo, P. (2019). Blackberry Crop Agroproductive Chain in El Altar Parish, Chimborazo-Ecuador. KnE Engineerin, 5(2), 576-596. doi: 10.18502/keg.v5i2.6280

Hernández, P. O. (2021). El cambio climático y las implosiones del ser humano. Con-Ciencia Serrana, 3(5), 11-14.

IPCC. (2015). Cambio climático 2014: Informe de síntesis. Contribución de los Grupos de trabajo I, II y III al Quinto Informe de Evaluación del Grupo Intergubernamental de Expertos sobre el Cambio Climático. Ginebra: OMM, PNUMA.

Izurieta, C. M., Atiencia, A. E., Fernández, V. D., \& Avalos, P. V. (2018). Análisis de la incidencia económica del trabajo infantil en el Cantón Riobamba. Revista Observatorio de la Economía Latinoamericana, 1-18.

La Frenierre, J., \& Mark, B. G. (2017). Detecting Patterns of Climate Change at Volcán Chimborazo, Ecuador, by Integrating Instrumental Data, Public Observations, and Glacier Change Analysis. Annals of the American Association of Geographers, 107(4), 979-997. doi:https://doi.org/10.1080/24694452.2016.1270185

Logroño-Rodríguez, M. F., Yumisaca-Jimenez, E., López-Calle, W., \& Flores-Pulgar, T. F. (2020). Contextualization of Agriculture and Heritagein order to Identify and Characterize Productsof Patrimonial Interest. KnE Engineering, 1, 674-696. doi:10.18502/keg.v5i2.6290

MAE. (2017). Tercera Comunicación Nacional del Ecua-dor a la Convención Marco de las Naciones Unidassobre el Cambio Climático. Quito: Dirección Nacional de Mitigación al Cambio Climático (DNMCC); Dirección Nacional de Adaptación al Cambio Climático (DNACC). 
MAE, M. d. (2016). Estrategia Nacional de Biodiversidad 2015-2030, primera edición. Quito: Ministerio del Ambiente del Ecuador. Obtenido de https://www.undp.org/content/dam/ecuador/docs/documentos\%20proyectos\%20am biente/Estrategia\%20Nacional\%20de\%20Biodiversidad\%202015-2030\%20\%20CALIDAD\%20WEB.pdf

MAE, M. d. (2019). Primera contribución determinada a nivel nacional para el Acuerdo de París bajo la Convención marco de Naciones Unidassobre cambio climático. Quito: Ministerio del Ambiente de Ecuador.

Martínez-Moscoso, A. (2019). El nuevo marco jurídico en materia ambiental en Ecuador. Estudio sobre el Código Orgánico del Ambiente. Actualidad Jurídica Ambiental, 89, $1-32$.

Mejía, P. M., Mejía, P. V., Veloz, M. S., \& Muquinche, U. M. (2019). El microcrédito como facilitador de empoderamiento. Experiencias de mujeres del sector rural de Chimborazo. La Ciencia al Servicio de la Salud y Nutrición, CSSN, 10(Ed. Esp), 353361.

Michel, F. (2020). Cambio climático y resilencia tradicional/ancestral: pueblos y nacionalidades indígenas del centro oriental de la Amazonía Ecuatoriana. Perspectivas: Revista de Historia, Geografía, Arte y Cultura , 8(15), 13-61.

Muyulema, A. J. (2018). La ecología industrial y la economía circular. Retos actuales al desarrollo de industrias básicas en el Ecuador. Dilemas Contemporáneos: Educación, Política y Valores, 5(2 Art. 53), 1-15. doi:https://doi.org/10.46377/dilemas.v16i2.44

Muyulema-Allaica, J. C., Canga-Castillo, S. M., Pucha-Medina, P. M., \& Espinosa-Ruiz, C. G. (2019). Evaluación de la contaminación por metales pesados en suelos de la Reserva Ecológica de Manglares Cayapas Mataje (REMACAM) - Ecuador. Revista Internacional de Investigación e Innovación Tecnológica - RIIIT, 7(41), 1-22.

Muyulema-Allaica, J. C., Pucha-Medina, P. M., Espinosa-Ruiz, C. G., \& Urquizo-Tenesaca, B. (2019). Reflexiones sobre la política exterior ecuatoriana en el marco de la globalización. Revista Publicando,, 6(21), 8-22.

OIT, O. I. (2018). Los pueblos indígenas y el cambio climático. De víctimas a agentes del cambiopor medio del trabajo decente. Ginebra : Oficina Internacional de Trabajo ILO, , \} Servicio de Género, Igualdad y Diversidad.

Peralta, L. O., Mejía, M. C., \& Gavidia, M. A. (2020). Condiciones de soberanía alimentaria en parroquias rurales de Chimborazo-Ecuador. Revista Espacios, 41(19), 411-425. 
Pérez, B. F. (2018). Migraciones climáticas: una aproximación al panorama actua. Tarragona: ECODES.

Portador-García, T. d. (2020). Los retos de la seguridad humana frente al cambio climático. Relaciones Internacionales, 43(1), 189 - 20.

Sánchez-Macías, R. A., Pucha-Medina, P. M., Usca-Veloz, R. B., Espinosa-Ruiz, C. G., Velasteguí-Bósquez, G. A., \& Muyulema-Allaica, J. C. (2020). Las finanzas sostenibles. Retos actuales hacia el desarrollo del sector cooperativo popular y solidario ecuatoriano. Revista Internacional de Investigación e Innovación Tecnológica-RIIIT. 7(42), 1-21.

Sandoval-Díaz, J., Cuadra-Martínez, D., Orellana-Fonseca, C., \& Sandoval-Obando, E. (2021). Diagnóstico comunitario ante desastres climáticos: Una experiencia de aprendizaje-servicio. ALTERIDAD. Revista de Educación, 16(1), 23-37. doi:https://doi.org/10.17163/alt.v16n1.2021.02

Toulkeridis, T., Tamayo, E., Simón-Baile, D., Merizalde-Mora, M. J., Reyes-Yunga, D. F., Viera-Torres, M., \& Heredia, M. (2020). Cambio Climático según los académicos ecuatorianos - Percepciones versus hechos. LA GRANJA. Revista de Ciencias de la Vida, 31(3), 21-46. doi:http://doi.org/10.17163/lgr.n31.2020.02

Tuaza, L. A. (2019). Desarrollo, identidad y poder en las comunidades indígenas de Chimborazo (Ecuador). Revista Andaluza de Antropología, 1(17), 11-30. doi:http://dx.doi.org/10.12795/RAA.2019.17.01

Tuaza, L. A., Jonhson, C., \& McBurney, M. (2020). Comunidad indígena de San Rafael de Chuquipogio, Chimborazo: transformacionesagrarias y cambio climático. Revista de Historia, Patrimonio, Arqueología y Antropología America - Rehpa, 3(1), 57-74. doi:https://doi.org/10.5281/zenodo.406576

Urquizo-Tenesaca, B., \& Muyulema-Allaica, J. C. (2019). Inmigración y Estado de bienestar. Una aproximación al caso ecuatoriano. Revista Dilemas Contemporáneos: Educación, Política y Valores, VI(2 Art. 43), 1-29. doi:https://doi.org/10.46377/dilemas.v22i2.600

Varea, M. S. (2020). Políticas públicas alrededor de loscuerpos de mujeres indígenas en laprovincia de Chimborazo en Ecuador. Estado \& comunes, Revista de políticas y problemas públicos, 1(2), 79-98. doi:https://orcid.org/0000-0002-3309-4977

Vázquez, R. B. (2020). El cambio climático en la agenda de seguridad de la Unión Europea. Relaciones Internacionales,

58 , 165-178. doi:https://doi.org/10.24215/23142766e086 
Vol. 4, $\mathrm{N}^{\circ} 1.2$, p. 470-488, marzo, 2021

Vivar, A. M., \& Sánchez, V. L. (2020). Evaluación ex-post proyecto quinua y su impacto socioeconómico en las familias asociadas, Chimborazo - Ecuador. Revista de Investigación de Agroproducción Sustentable, 4(1), e536. doi:http://dx.doi.org/10.25127/aps.20201.536 


\section{PARA CITAR EL ARTÍCULO INDEXADO.}

Bolívar Urquizo , T. (2021). Cambio climático y migración en los pueblos indígenas de la provincia de Chimborazo - Ecuador . ConcienciaDigital, 4(1.2), 470-488. https://doi.org/10.33262/concienciadigital.v4i1.2.1623

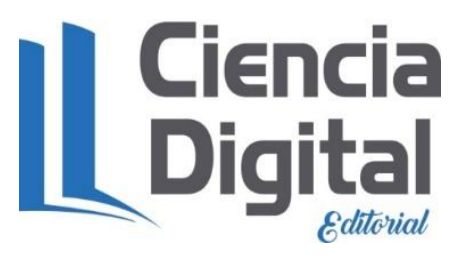

El artículo que se publica es de exclusiva responsabilidad de los autores y no necesariamente reflejan el pensamiento de la Revista Conciencia Digital.

El artículo queda en propiedad de la revista y, por tanto, su publicación parcial y/o total en otro medio tiene que ser autorizado por el director de la Revista Conciencia Digital.
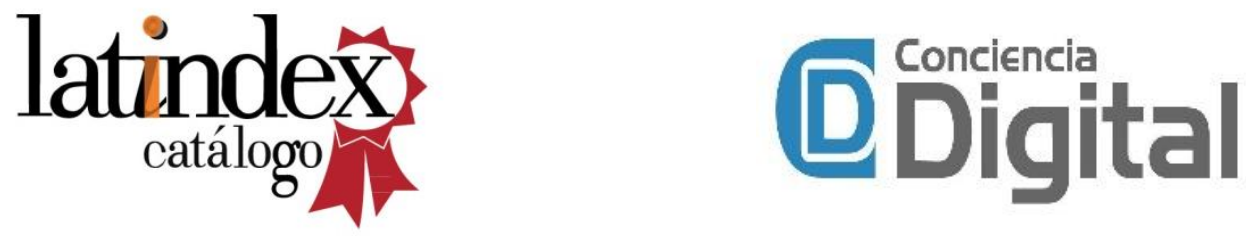